\title{
Book Review: Christian Reichardt and Thomas Welton, Solvents and Solvent Effects in Organic Chemistry (Fourth Edition, Updated and Enlarged; Wiley-VCH Verlag \& Co. KGaA, Weinheim, 2011; 718 pages. Hardcover)
}

DOI: $10.1134 / \mathrm{S} 0036024411080231$

The diversity of mainly organic solvents is opening increasingly broader opportunities for managing chemical and physicochemical processes. This is demonstrated with particular cogency by the fourth edition of Solvents and Solvent Effects in Organic Chemistry by the well-known German chemist Christian Reichardt, published at the beginning of this year in coauthorship with his British colleague Thomas Welton. The third edition of the book, issued in 2003, quickly sold out and printed twice more in 2004 and 2005. The rapid expansion of the number of researchers in the field of liquid-phase reaction fueled the need for a new edition, posing the authors with a difficult task: the soft revolution that has taken place in chemistry in recent years has made it impossible to simply review or even mention all of the works in the field of solutions. Nevertheless, the authors have managed to combine completeness of coverage of theoretical problems with the reference character of the book, having largely retained their encyclopedic style of presentation.

The present edition has been expanded by approximately $15 \%$ over with the earlier work (see Zhurn. Fiz. Khim., 2004, vol. 78, no. 2, p. 383), for which it was necessary to sacrifice the authors' index while keeping only the subject index. Each section has been supplemented with information reflecting the results from new publications, including reviews (up to 2010, inclusive) and supplied with the appropriate bibliographic references (now more than 4000 in number, approximately $20 \%$ more than in the third edition of the book). After an interesting historical introduction, the book begins with detailed and qualified consideration of the problem of solute-solvent interaction. Later, it discusses the classification of existing solvents. The next two chapters offer an extensive review of dif- ferent aspects of the effect exerted by a solvent on chemical equilibria and particularly on the rates of homogenous chemical reactions. The information on the effect exerted by the nature of a solvent on the electronic absorption and fluorescence spectra, and on the IR-, NMR-, and EPR-spectra of circular dichroism, is presented systematically.

The sections devoted to solvatochromism are valuable in themselves (Prof. Reichardt is a recognized world leader in this field) and prepare the reader for subsequent consideration of the versatile empirical parameters of the polarity of solvents. This edition of the book contains a new chapter, "Solvents and Green Chemistry," devoted to the field in which Prof. Welton is expert. This chapter considers alternative solvents (e.g., supercritical carbon dioxide, low-temperature ionic liquids, and other liquid systems recently investigated), their industrial application, and classifying solvents according to their effect on the environment.

The appendix, which contains data on the physical properties and purification of solvents and groups the information on separate aspects of their application (in spectroscopy, electrochemistry, chromatography, extraction, acid-base titration, etc.) is a valuable tool for researchers working in different fields of chemistry. The concluding chapter provides information on the toxicity of organic compounds.

On the whole, we can agree with the opinion of Wiley-VCH that no other book now offers such exhaustive and deep information on the influence of solvents on chemical processes. This work is sure to be useful for all researchers, graduate students, and undergraduates working with liquid media.

N.O. Mchedlov-Petrosyan 